\title{
ASYMMETRIC DRIVING BEHAVIOUR ANALYSIS USING FIELD TRAJECTORIES
}

\author{
XIULING HUANG, JIE SUN \& JIAN SUN \\ Department of Traffic Engineering and Key Laboratory of Road and Traffic Engineering, \\ Ministry of Education, Tongji University, China
}

\begin{abstract}
Asymmetric driving behaviour in acceleration and deceleration processes under car-following (CF) state can affect traffic flow significantly. Thus, to improve the performance of CF models, the formation mechanism of the asymmetric driving characteristics (gap difference, reaction time difference, response intensity difference, discrete driving difference) and the internal relations between them should be investigated. Thus the quantification methods are proposed to study the asymmetry for each driving characteristic. Then the mean values of the gap, reaction time, discrete intensity, and response intensity at each velocity fragment in acceleration and deceleration process are measured respectively using the NGSIM data with the proposed quantification methods. By comparing these mean values in acceleration and in deceleration processes, the asymmetry of the gap, reaction time, discrete intensity and response intensity are validated. Moreover, the correlation analysis between the four asymmetric driving characteristics are implemented while the results indicate they influence each other, and the formation mechanisms of the four asymmetric driving behaviours are obtained.

Keywords: car-following, asymmetric driving behaviour, formation mechanism, quantification methods, correlation analysis.
\end{abstract}

\section{INTRODUCTION}

Asymmetric driving behaviour possessing gap difference, reaction time (RT) difference, discrete driving (DD) difference, response intensity (RI) difference characteristic in accelerating and decelerating process under the car-following (CF) condition is reported by many researchers [1]-[3], which can affect the traffic flow significantly [4]. Thus, the performance of $\mathrm{CF}$ models in reproducing the asymmetric driving behaviour affects the numerical simulation results [5]. Many CF models [6], [7] based on simple assumptions cannot capture the realistic traffic flow characteristics as well as that considering asymmetric driving behaviour [3], [5], [8]-[11]. For instance, the asymmetry full velocity difference (AFVD) model proposed by Gong et al. [9] which considers the gap difference and RI difference outperforms the optimal velocity model (OVM) proposed by Bando et al. [7] without considering the asymmetric driving behaviour in terms of reproducing the realistic traffic flow characteristics.

In addition, some studies indicated that the asymmetric driving characteristics affect each other [2], [6], [12]-[15]. Specifically, both Helly [6] and Newell [13] believed that the gap is influenced by RT. In consideration of the RT difference between acceleration and deceleration process [14], [15], the gap in acceleration and deceleration process should be different as well. Hidas [16] believed that the asymmetric driving behaviours are only formed in CF condition rather than in free flow condition. Yeo [2] also proposed two different gap functions for acceleration and deceleration process, both in which, the gap increases with RT increasing. Besides, Yeo [2] put forward that the DD in deceleration process are more frequent which leads to the short RT. However, this theory is not proved by the field data. Lenz et al. [17] also proposed a CF model based on the assumption that the acceleration increase with larger gap. However, little effort has been devoted to understanding the relationships between the characteristics of asymmetric driving behaviour. 
To prove the internal relations between the asymmetric driving behaviours in depth and obtain the mechanism of the asymmetric driving behaviours with field data, the remaining parts are organized as follows: Section 2 describes the data preparation process; Section 3 analyses the four asymmetric driving characteristics with the quantified methodologies; Section 4 implements the correlation analysis to obtain the effects between the four characteristics; Section 5 summarizes main conclusions of this study.

\section{DATA}

The datasets used in this study are collected from two study sites from the Next Generation Simulation (NGSIM) program [18], i.e. the U.S. Highway 101 in Los Angeles, California and the Interstate 80 in Emeryville, California. In these datasets the observed data are collected every $0.1 \mathrm{~s}$. According to Montanino and Punzo [19], the original datasets contain abnormal values of acceleration and speed. Therefore, Kalman filtering [20] is used for reducing the measurement errors in this paper. In addition, to obtain the appropriate data, the trajectory data used in this study need be further filtered according to the following conditions:

(1) Each leader-follower trajectory pair should keep driving on the same lane without any lane-changing behaviour;

(2) The speed should be limited in $[3 \mathrm{~m} / \mathrm{s}, 15 \mathrm{~m} / \mathrm{s}]$, and the gap between the leader and follower should be smaller than $50 \mathrm{~m} \mathrm{[21]}$;

(3) The trajectories with $\left|a_{j}^{i}\right| \geq 0.3 \mathrm{~m} / \mathrm{s}^{2}$ are regarded as acceleration/deceleration data [2];

(4) Each acceleration/deceleration process should last for more than $6 \mathrm{~s}$, containing only accelerating/decelerating and coasting behaviours.

\section{QUANTIFIED METHODOLOGIES AND EMPIRICAL ANALYSIS}

To understand the mechanism of asymmetric driving behaviour and the relationship between the asymmetric characteristics from the filed data, the driving characteristics are defined and quantified accurately in this section.

\subsection{Gap difference}

The gap in this study is defined as the net distance gap between the rear bumper of the leader and the front bumper of the follower. The speed-gap relationship of vehicle 1212 in Fig. 1 indicates that the gap in acceleration is different from the gap in deceleration when driving at the same velocity. In other words, the relationship during accelerating and decelerating is asymmetric. This feature exists universally under CF condition.

To prove this feature, the acceleration and deceleration trajectories are organized for 13 segments respectively based on the velocity range according to the condition (2) in Section 2 (see Fig. 2). Each $1 \mathrm{~m} / \mathrm{s}$ increase in velocity is considered as an individual velocity fragment (i.e. $3 \mathrm{~m} / \mathrm{s}, 4 \mathrm{~m} / \mathrm{s}, 5 \mathrm{~m} / \mathrm{s})$. Thus, the average gap for acceleration $\left(\overline{g a p_{v l}^{a}}\right)$ and deceleration $\left(\overline{g a p_{v l}^{d}}\right)$ process at each velocity fragment $(v i=3 \mathrm{~m} / \mathrm{s}, 4 \mathrm{~m} / \mathrm{s}, \ldots 15 \mathrm{~m} / \mathrm{s}$ can be obtained respectively. The gap difference $\left(\overline{g a p_{v l}^{d l f f}}\right)$ at each velocity fragment is then calculated with eqn (1). The overall average gap for acceleration $\left(\frac{1}{13} \sum_{v i=3}^{15} \overline{g^{a p_{v l}^{a}}}\right)$ and deceleration $\left(\frac{1}{13} \sum_{v i=3}^{15} \overline{g a p_{v l}^{d}}\right)$ process within all velocity fragments are also obtained, and the corresponding average gap difference $\left(\overline{g a p_{-} d l f f}\right)$ is then calculated with eqn (2). According to these quantified methods, values of these variables are obtained and shown in Table 1: 


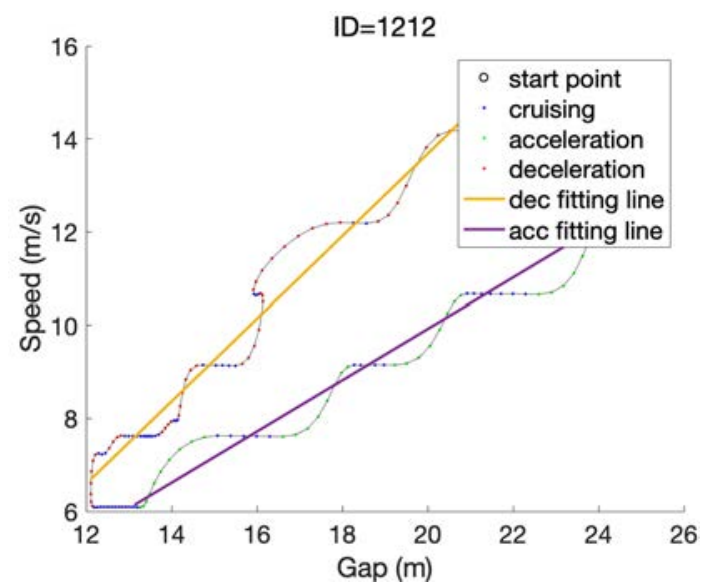

Figure 1: Gap difference in acceleration and deceleration process.

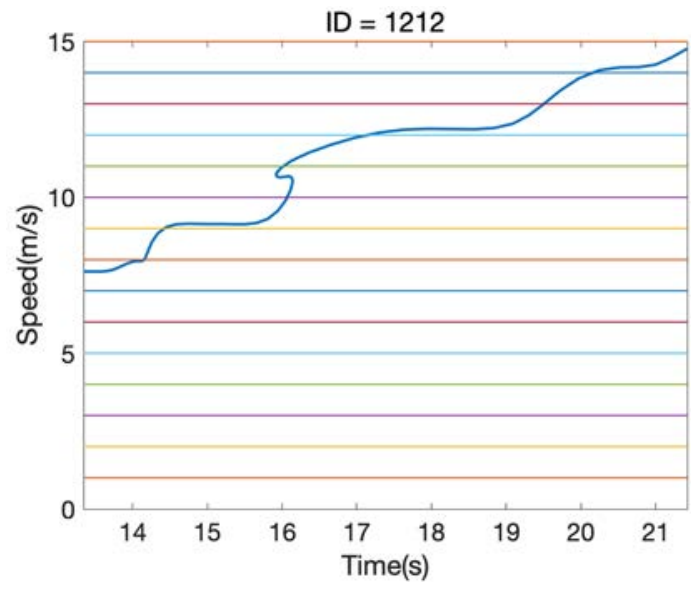

Figure 2: Quantified method for gap difference.

$$
\begin{gathered}
\overline{g a p_{v l}^{d l f f}}=\overline{g a p_{v l}^{a}}-\overline{g a p_{v l}^{d}}, \\
\overline{g a p_{-} d l f f}=\frac{1}{13} \sum_{v i=3}^{15} \overline{g a p_{v l}^{a}}-\frac{1}{13} \sum_{v i=3}^{15} \overline{g a p_{v l}^{d}} .
\end{gathered}
$$

From Table 1 we can see that the $\overline{g a p_{v l}^{a}}$ is always lager than $\overline{g a p_{v l}^{d}}$ when $v \leq 13 \mathrm{~m} / \mathrm{s}$. In these cases, each $\overline{g a p_{v l}^{d l f f}}$ is larger than $3.8 \mathrm{~m}$, which indicates that the gap difference is obvious between acceleration and deceleration process. However, the $\overline{g a p_{v l}^{a}}$ is close but a little smaller than $\overline{g a p_{v l}^{d}}$ when $v=14 \mathrm{~m} / \mathrm{s}$. And $\overline{g a p_{a}}(15)$ does not exist since there is no trajectory data. The $\overline{g a p_{-} d l f f}=4.79 \mathrm{~m}$ is larger more than zero. Therefore, Table 1 implies that the relation between acceleration gap and deceleration gap is asymmetry. 
Table 1: Summary of average gap and gap difference.

\begin{tabular}{|c|c|c|c|c|c|c|c|}
\hline $\begin{array}{c}\text { Speed } \\
(\mathrm{m} / \mathrm{s})\end{array}$ & $\begin{array}{c}\overline{g a p_{v l}^{d}} \\
(\mathrm{~m})\end{array}$ & $\begin{array}{c}\overline{g a p_{v l}^{a}} \\
(\mathrm{~m})\end{array}$ & $\begin{array}{c}\overline{g a p_{v l}^{d l f f}} \\
(\mathrm{~m})\end{array}$ & $\begin{array}{c}\text { Speed } \\
(\mathrm{m} / \mathrm{s})\end{array}$ & $\begin{array}{c}\overline{g a p_{v l}^{d}} \\
(\mathrm{~m})\end{array}$ & $\begin{array}{c}\overline{g a p_{v l}^{a}} \\
(\mathrm{~m})\end{array}$ & $\begin{array}{c}\overline{g a p_{v l}^{d l f f}} \\
(\mathrm{~m})\end{array}$ \\
\hline 3 & 6.37 & 10.39 & 4.01 & 10 & 14.15 & 20.12 & 5.97 \\
\hline 4 & 7.36 & 11.52 & 4.17 & 11 & 16.11 & 21.67 & 5.56 \\
\hline 5 & 9.08 & 12.91 & 3.83 & 12 & 16.07 & 22.92 & 5.85 \\
\hline 6 & 9.93 & 14.28 & 4.35 & 13 & 17.16 & 24.83 & 7.67 \\
\hline 7 & 10.44 & 15.84 & 5.41 & 14 & 20.52 & 20.14 & -0.38 \\
\hline 8 & 12.20 & 17.04 & 4.84 & 15 & 21.65 & - & - \\
\hline 9 & 13.39 & 18.59 & 5.20 & total & 12.73 & 17.52 & 4.79 \\
\hline
\end{tabular}

\subsection{Reaction time difference}

Many methods for estimating the RT have been proposed based on the theory that the subject vehicle always changes driving behaviour some time later than the leader [13]-[15], [23], [24]. However, among these methods, some are laborious and time consuming, such as the RT defined in Zheng et al. [23] is the time lag between the starting points of gap and speed variation of the subject vehicle; the RT defined in Khodayari et al. [24] is the time lag between the starting points of relative speed and acceleration of the subject vehicle.

To prove the RT difference between acceleration and deceleration process, three methods that can estimate the RT more convenient are used in this study. Method I: the method in [14]. Ozaki proposed that the RT is a function of gap and the acceleration of the leader. Method II: the method in [13]. The definition of RT in [13] is that the follower (vehicle $i$ ) changes velocity following to the lead vehicle's velocity changed after $\tau$ s. For instance, the vehicle $i-1$ drives at constant velocity $v$ for a period of time, and then changes to constant velocity $v^{\prime}$. The follower will do the similar change with a space and time placement $d_{i}$ and $\tau_{i}$. According to this theory, Newell [13] proposed that the distance between the leading and following vehicles is relative to $d_{i}$ and $\tau_{i}$, and the relationship of them can refer to the [13]. Method III: the method in [15]. Siuhi proposed that there is a linear relationship between relative speed at time $t(\Delta v(t))$ and the acceleration/deceleration at time $t+\Delta t(a(t+\Delta t))$ and the RT is the time lag $(\Delta t)$ when the goodness of fit (R-square) is the highest between the field data and the fitting function.

In order to remove the noise trajectories, the pre-processed data need to be filtered according to [22]. To estimate the RT with Method II and Method III, Least Square Method (LSM) is used, with the range of $d_{i} \in[0.1 \mathrm{~m}, 50 \mathrm{~m}]$. For $\tau_{i}$, the low limit set as $-1 \mathrm{~s}$ represents considering multi-leaders, and the upper limit is set as $5.0 s$ [23]. The negative value of $\tau_{i}$ represents that the follower perceives the driving behaviour from vehicles not only the immediate leader. The RT in acceleration and deceleration process estimated from these three methods are summarized in Table 2.

From Table 2, we can see that the RT between the acceleration and deceleration process are different. The mean RT of method I, II and III in deceleration are 7.1\%, 49.25\% and $11.79 \%$ shorter than that in acceleration respectively. Both the minimum RT that calculated from [13] and [15] are negative, which can indicate some drivers anticipate the driving behaviour from multi-leaders. Both the average RTs in acceleration and deceleration process estimated from [15] are longer than $2 \mathrm{~s}$, which seems inconsistent with the facts. For Method 
Table 2: Reaction time with different methods.

\begin{tabular}{|l|c|c|c|c|c|}
\hline Model & & Mean (s) & Std. (s) & Max (s) & Min (s) \\
\hline \multirow{2}{*}{ Ozaki (1993) } & acc & 1.36 & 0.22 & 2.05 & 0.80 \\
\cline { 2 - 6 } & dec & 1.27 & 0.24 & 2.07 & 0.75 \\
\hline \multirow{2}{*}{ Newell (2002) } & acc & 1.0 & 0.98 & 4.35 & -1.00 \\
\cline { 2 - 6 } & dec & 0.67 & 0.88 & 3.89 & -1.00 \\
\hline \multirow{2}{*}{$\begin{array}{l}\text { Newell (2002) without } \\
\text { negative RT }\end{array}$} & acc & 1.33 & 0.69 & 4.35 & 0.00 \\
\cline { 2 - 6 } & dec & 0.99 & 0.65 & 3.89 & 0.00 \\
\hline \multirow{2}{*}{ Siuhi (2009) } & acc & 2.37 & 1.96 & 5.0 & -1.0 \\
\cline { 2 - 6 } & dec & 2.12 & 1.94 & 5.0 & -1.0 \\
\hline
\end{tabular}

I, though they believed that $r t$ in acceleration is different from that in deceleration process, the RT in acceleration is very close to that in deceleration (the RT difference is smaller than $0.1 \mathrm{~s}$ ) and both the maximum RT in acceleration and deceleration are approximately equal to $2 \mathrm{~s}$, all of which seem inconsistent with the facts as well. Thus, the $r t$ that estimated from Method II is used in the following sections.

\subsection{Discrete driving difference}

Yeo [2] pointed out that the follower usually assesses the spacing and speed every certain seconds when accelerating or decelerating, which leads to accelerating or decelerating discretely. As shown in Fig. 3, the acceleration curve is not straight. Since different drivers have difference DD frequencies during the same period, such as $6 \mathrm{~s}$, to quantify the intensity of $\mathrm{DD}$, the variable named discrete intensity (DI) is introduced to measure the DD frequency. A complete DD should satisfy the following conditions: (1) the acceleration/deceleration trajectory should include a short acceleration/deceleration and short cruising driving and start from accelerating/decelerating, (2) the driving behaviour that $\left|a_{j}^{i}\right|<0.3 \mathrm{~m} / \mathrm{s}^{2}$ is regarded as cruising driving, (3) the time of a continuous acceleration/ deceleration should be larger than $0.2 \mathrm{~s},(4)$ the time of continuous cruising driving should be larger than $0.2 \mathrm{~s}$ and smaller than the cruising threshold time (ctt). In this study, ctt is set as $1 \mathrm{~s}$.

Therefore, the DI of acceleration and deceleration are estimated and summarized in Table 3. From Table 3, we can see that the DI in acceleration and deceleration process are different and the DI in acceleration is $14.06 \%$ larger than that in deceleration.

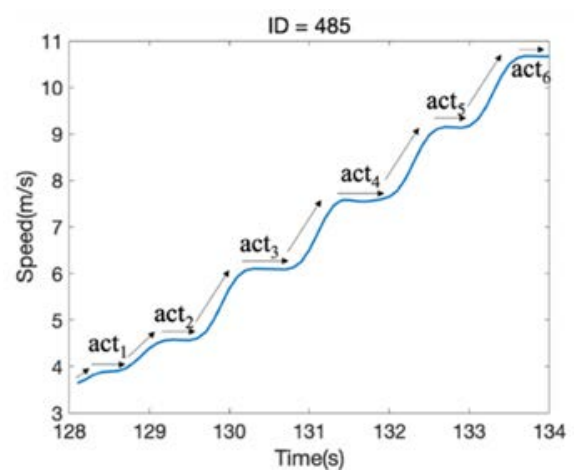

Figure 3: Illustration of discrete driving. 
Table 3: Discrete intensity in acceleration and deceleration process.

\begin{tabular}{|c|c|c|c|c|}
\hline \multicolumn{2}{|c|}{ Acceleration } & \multicolumn{2}{c|}{ Deceleration } & \\
\hline Mean (1/s) & Std. (1/s) & Mean (1/s) & Std. (1/s) & Difference \\
\hline 2.19 & 1.21 & 1.92 & 1.05 & $14.06 \%$ \\
\hline
\end{tabular}

\subsection{Response intensity difference}

Many studies have proved that the RI of drivers (usually measured by acceleration) to the same-magnitude relative speed (could be positive or negative) are different, when driving in the same traffic condition (i.e. the same velocity and the same distance gap) [3]. Thus, the RI at time $t$ and at certain velocity $(v i)$ and gap $($ gapi $)$ is defined as rint $t_{v i, g a p i, t}=$ $a_{v i, g a p i, t} / \Delta v_{v i, g a p i, t}$ at in this study. In $a$ Note that small acceleration or relative speed may be caused by the driving error or reaction delay, which may lead to RI noise, e.g. due to perception error, the follower maintains accelerating/decelerating when the speed has been approximately equal to the leader's, which results in small $\Delta v$ (e.g. $\Delta v<0.06 \mathrm{~m} / \mathrm{s}$ ) and large RI that can't represent the true RI of the follower. Therefore, the trajectories used for quantifying should satisfy the following conditions: (1) $\left|a_{j}^{i}\right| \geq 0.3 \mathrm{~m} / \mathrm{s}^{2}$, (2) $|\Delta v| \geq \Delta V$. The range of the $\Delta V$ can be set as $[0.03 \mathrm{~m} / \mathrm{s}, 0.15 \mathrm{~m} / \mathrm{s}]$ base on our experiments. Both the acceleration and deceleration datasets are organized as $13 \times 23$ segments. Each $1 \mathrm{~m} / \mathrm{s}$ increase in velocity is considered as an individual velocity segment (from $3 \mathrm{~m} / \mathrm{s}$ to $15 \mathrm{~m} / \mathrm{s}$ ) and each gap segment increase $2 \mathrm{~m}$ from $5 \mathrm{~m}$ to $50 \mathrm{~m}$ at each velocity fragment. Thus there are 299 acceleration data subsets and 299 deceleration data subsets. And the average RI at certain speed (vi) and certain gap (gapi) in acceleration $\left(\right.$ rint $\left._{v i, g a p i}^{a}\right)$ and deceleration (rint $\left.t_{v i, g a p i}\right)$ can be estimated with eqn (3) and eqn (4) respectively with $\Delta V=0.06 \mathrm{~m} / \mathrm{s}$. The mean RI at certain velocity fragment including all distance fragments for acceleration $\left(\operatorname{rint}_{v i}^{a}\right)$ and deceleration $\left(r i n t_{v i}^{d}\right)$ is calculated from eqns (5) and (6). Therefore, the overall average RI including all velocity fragments and gap fragments in acceleration $\left(\operatorname{rint}^{a}=\operatorname{mean}\left(\operatorname{rint}_{v i}^{d}\right)\right)$ and deceleration $\left(\operatorname{rint}^{d}=\operatorname{mean}\left(\operatorname{rint}_{v i}^{d}\right)\right)$ can be obtained. The results are shown in Table 4.

$$
\begin{aligned}
& \operatorname{rint}_{v i, g a p i}^{a}=\frac{1}{n a} \sum_{i=1}^{n a} \frac{a_{v i, g a p i, t}^{i}}{\Delta v_{v i, g a p i, t}^{a, i}} \\
& \operatorname{rint}_{v i, g a p i}^{d}=\frac{1}{n d} \sum_{i=1}^{n d} \frac{d_{v i, g a p i}^{i}}{\Delta v_{v i, g a p i}^{d, i}} \\
& \operatorname{rint}_{v i}^{a}=\frac{1}{23} \sum_{g a p i=5}^{49} \operatorname{rint}_{v i, g a p i}^{a}, \\
& \operatorname{rint}_{v i}^{d}=\frac{1}{23} \sum_{g a p i=5}^{49} \operatorname{rint}_{v i, g a p i}^{d},
\end{aligned}
$$

where $a_{v i, g a p i}^{i}, d_{v i, g a p i}^{i}$ represent the acceleration and deceleration when driving at $v i \mathrm{~m} / \mathrm{s}$ and gapi $m . \Delta v_{v i, g a p i}^{a, i}, \Delta v_{v i, g a p i}^{d, i}$ represents the relative speed in acceleration and deceleration when driving at $v i \mathrm{~m} / \mathrm{s}$ and gapi $m$. $i$ represents the index of trajectory record. na and $n d$ represent the size of each acceleration and deceleration data subset. 
Table 4: Response intensity in acceleration and deceleration process.

\begin{tabular}{|c|c|c|c|c|c|}
\hline \multirow{2}{*}{$\begin{array}{l}\text { Speed } \\
(\mathrm{m} / \mathrm{s})\end{array}$} & Acceleration & Deceleration & \multirow{2}{*}{$\begin{array}{c}\text { Speed } \\
(\mathrm{m} / \mathrm{s})\end{array}$} & Acceleration & Deceleration \\
\hline & $\operatorname{rint}_{v i}^{a}(1 / \mathrm{s})$ & $\operatorname{rint}_{v i}^{d}(1 / \mathrm{s})$ & & $\operatorname{rint}_{v i}^{a}(1 / \mathrm{s})$ & $\operatorname{rint}_{v i}^{d}(1 / \mathrm{s})$ \\
\hline 3 & 0.88 & 1.64 & 10 & 3.48 & 3.23 \\
\hline 4 & 3.60 & 6.19 & 11 & 3.07 & 3.18 \\
\hline 5 & 3.51 & 8.75 & 12 & 4.92 & 1.46 \\
\hline 6 & 1.47 & 1.32 & 13 & 3.84 & 2.93 \\
\hline 7 & 4.09 & 3.75 & 14 & $\mathrm{NaN}$ & 1.53 \\
\hline 8 & 2.29 & 4.42 & 15 & $\mathrm{NaN}$ & 0.58 \\
\hline 9 & 2.26 & 1.34 & Total & 2.67 & 3.20 \\
\hline
\end{tabular}

From Table 4, we can see that (1) the relationship of RI between acceleration and deceleration is asymmetric at each speed fragment, except $v i=14 \mathrm{~m} / \mathrm{s}$ and $v i=15 \mathrm{~m} / \mathrm{s}$ while there are no data for $\operatorname{rint}_{14}^{a}$ and $\operatorname{rint}_{15}^{a}$; (2) the $\operatorname{rint}_{v i}^{a}$ s are not always smaller than $\operatorname{rint}_{v i}^{d} s$. For instance, when driving at low speeds, such as speeds lower than $9 \mathrm{~m} / \mathrm{s}$, most magnitudes of RI in acceleration are smaller than that in deceleration. However, when driving at the higher speed, such as speeds greater than $9 \mathrm{~m} / \mathrm{s}$, most magnitudes of RI in acceleration are larger than that in deceleration. This result seems not consistent with the theory of RI asymmetry which is believed by previous researchers [9], [10]. In order to prove this result in depth, the magnitudes of RI at certain speed and gap in acceleration and deceleration are compared. The results show that when driving at high speeds (e.g. $12 \mathrm{~m} / \mathrm{s})$, the most magnitudes of rint ${ }_{v i, g a p i}^{a} \mathrm{~s}$ are higher than rint $_{v i, g a p i}^{d} \mathrm{~s}$ (see Fig. 4(a)). When driving at low speeds (e.g. $5 \mathrm{~m} / \mathrm{s}$ ), each magnitude of rint ${ }_{v i \text {,gapi }}^{a}$ is lower than rint ${ }_{v i, g a p i}^{d}$ (see Fig. 4(b)). Therefore, the RIs at certain speed and gap between acceleration and deceleration are asymmetry. Moreover, the magnitudes of RIs are not always higher in deceleration than that in acceleration when driving at the same traffic situation.

\section{MECHANISM OF THE ASYMMETRIC DRIVING BEHAVIOR}

The asymmetry in the gap, DD, RT and RI between acceleration and deceleration process have been proved in the Section 3. To obtain the formation mechanism of gap difference, the correlation analysis between the gap and $|\Delta g a p|$ and other three characteristics respectively are implemented and the results are shown in Tables 5-7 respectively. Note that the RTs that larger than zero are extracted and used. Each driver is considered as having one RT and DI.

As shown in Table 5, the RT has significant positive correlation with gap both in acceleration and deceleration. It means the gap increases with RT increasing both in acceleration and deceleration, vice versa. This results is consistent with the gap model proposed in [2], [6], [13]. In other words, drivers keep larger gap from the leader if his/her $\mathrm{RT}$ is longer. Thus, the larger RT in accelerating than in decelerating leads to the larger gap difference between acceleration and deceleration. Moreover, the $|\Delta g a p|$ have significant positive correlation with the RT both in acceleration and deceleration. In this way, the slopes of the gap-speed fitting lines for acceleration and deceleration are different, such as the fitting lines shown in Fig. 1.

As shown in Table 6, the correlation between gap and DI is significant in acceleration process, but not significant in deceleration process. However, the correlativity is very significant between $|\Delta g a p|$ and DI in both processes. Both the gap and $|\Delta g a p|$ increase with the increase of DI during the acceleration process. During the deceleration process, only 


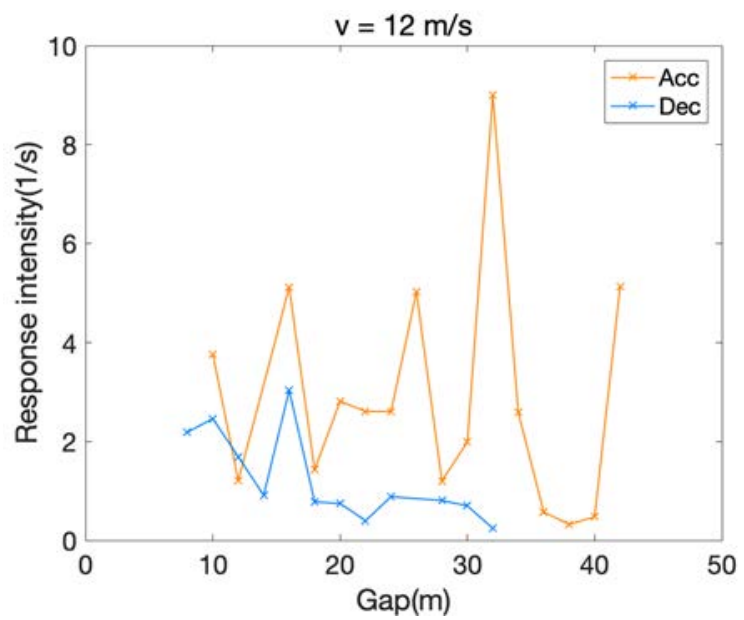

(a)

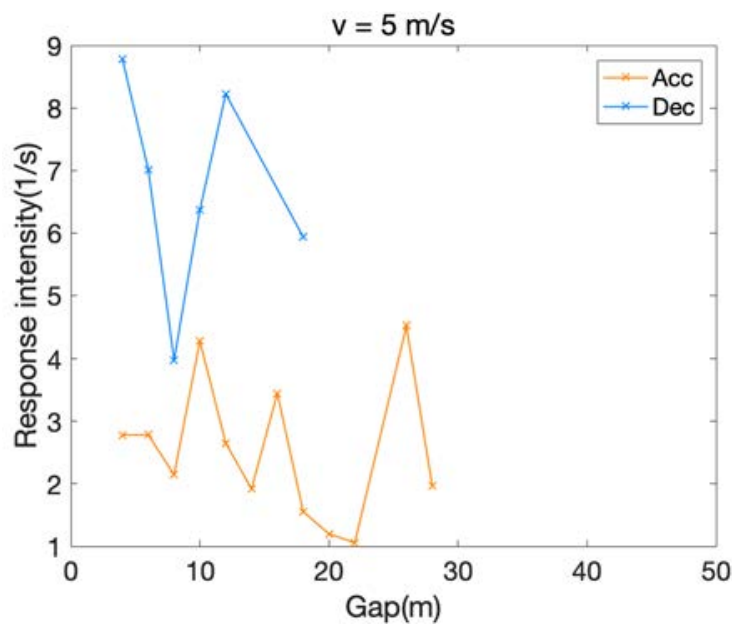

(b)

Figure 4: Response intensity at certain speed and gap. (a) $v=12 \mathrm{~m} / \mathrm{s}$; and (b) $v=5 \mathrm{~m} / \mathrm{s}$

Table 5: Correlation between the gap/Dgap and reaction time.

\begin{tabular}{|l|c|c|}
\hline & Acceleration & Deceleration \\
\hline Gap and reaction time $(R T \geq 0)$ & $0.435^{* *}(0.000)$ & $0.282^{* *}(0.000)$ \\
\hline$\Delta g a p$ and reaction time $(R T \geq 0)$ & $0.235^{* *}(0.000)$ & $0.222^{* *}(0.008)$ \\
\hline
\end{tabular}

Table 6: Correlation between gap/ $\Delta$ gap and discrete intensity.

\begin{tabular}{|l|c|c|}
\hline & Acceleration & Deceleration \\
\hline Gap and discrete intensity & $0.122^{*}(0.022)$ & $0.049(0.491)$ \\
\hline$\Delta$ gap and discrete intensity & $0.292^{* *}(0.000)$ & $0.218^{* *}(0.002)$ \\
\hline
\end{tabular}


Table 7: Correlation between the gap and response intensity.

\begin{tabular}{|c|c|c|c|c|c|}
\hline $\begin{array}{c}\text { Speed } \\
(\mathrm{m} / \mathrm{s})\end{array}$ & Acceleration & Deceleration & $\begin{array}{c}\text { Speed } \\
(\mathrm{m} / \mathrm{s})\end{array}$ & Acceleration & Deceleration \\
\hline 3 & $-0.264^{* *}(0.002)$ & $-0.449(0.225)$ & 10 & $-0.150(0.065)$ & $-0.123(0.193)$ \\
\hline 4 & $-0.198^{*}(0.049)$ & $-0.175(0.487)$ & 11 & $-0.114(0.240)$ & $-0.324^{* *}(0.001)$ \\
\hline 5 & $-0.169^{*}(0.019)$ & $-0.098(0.348)$ & 12 & $-0.191(0.119)$ & $-0.257^{*}(0.023)$ \\
\hline 6 & $-0.225^{* *}(0.000)$ & $-0.202^{*}(0.034)$ & 13 & $-0.299(0.091)$ & $-0.160(0.288)$ \\
\hline 7 & $-0.312^{* *}(0.000)$ & $-0.160(0.085)$ & 14 & 0 & $-0.182(0.326)$ \\
\hline 8 & $-0.274^{* *}(0.000)$ & $-0.034(0.704)$ & 15 & 0 & $-0.614(0.142)$ \\
\hline 9 & $-0.154^{*}(0.025)$ & $-0.275^{* *}(0.001)$ & total & $-0.102^{* *}(0.000)$ & $-0.184^{* *}(0.000)$ \\
\hline
\end{tabular}

$|\Delta g a p|$ increases with the increase of DD frequency. The relationship between $\Delta g a p$ and DI can be indicated by Fig. 5. In Fig. 5(a), the gap increases when discretely driving in acceleration process, though the magnitudes of each gap change are different. For instance, when discretely driving at $v_{1}^{\prime}$ and $v_{2}^{\prime}$, the gap increases $\Delta g a p_{1}^{\prime}$ and $\Delta g a p_{2}^{\prime}$ respectively with $\Delta g a p_{1}^{\prime}>\Delta g a p_{2}^{\prime}$. In Fig. 5(b), the gap decreases when discretely driving in the deceleration process though the magnitudes of each gap varying are different. For instance, the gap decreases $\Delta g a p_{1}^{\prime}$, and $\Delta g a p_{2}^{\prime}$, when DD at $v_{1}^{\prime}$ and $v_{2}^{\prime}$, respectively, and $\Delta g a p_{2}^{\prime}>\Delta g a p_{1}^{\prime}$.

Since RI is measured at certain speed and gap. The correlation between gap and RI is analysed based on each velocity fragment from $3 \mathrm{~m} / \mathrm{s}$ to $15 \mathrm{~m} / \mathrm{s}$ with $1 \mathrm{~m} / \mathrm{s}$ interval. Zero in Table 7 at $14 \mathrm{~m} / \mathrm{s}$ and $15 \mathrm{~m} / \mathrm{s}$ in acceleration represents not enough data.

From Table 7, we can see that the correlation between gap and RI are affected by the speed, in both acceleration and deceleration process. When speed is smaller than $10 \mathrm{~m} / \mathrm{s}$, correlation between gap and RI is significant and negative influence in accelerating. When speed is no smaller than $9 \mathrm{~m} / \mathrm{s}$, no significant correlation is observed between gap and RI. In deceleration, the correlation between gap and RI is significant and negative influence when driving at some speeds, such as $6 \mathrm{~m} / \mathrm{s}, 9 \mathrm{~m} / \mathrm{s}$. However, the correlation between gap and RI is significant and negative influence while including all trajectories.

Besides, to obtain the mechanism of the asymmetric driving behaviour, the correlation between RI, RT and DI are implemented as well. The relationship between RT, DI and RI are given in Table 8. From Table 8, we can see that the RT increase with the DI decrease in both acceleration and deceleration process. The smaller relative speed between the follower and leader also leads to less discretely driving. However, both the relationship between RI and RT, DI and RT are not significant. In other words, the RT of a driver does not impact the $\mathrm{RI}$ and DI. The reason for this result may be caused by the RI, since the RI here is the mean value for each driver, but not based on a certain gap and velocity.

Based on the above analysis, we can obtain some findings about the formation mechanism of the asymmetric driving behaviour: (1) The gap can be categorized as a basic gap which is influenced by RT mainly, and varying gap that is influenced by DI and RI. (2) In deceleration process, drivers pay more attention on safety, which leads to shorter RTs, smaller basic gaps, and smaller deceleration. In contrast to the deceleration process, there are longer RTs, larger basic gaps, and larger acceleration in acceleration process. (3) The smaller deceleration leads to larger real gap than desired gap. However, for safely driving, drivers usually do not reduce the gap with discretely driving, which results in low DI in deceleration process. In acceleration process, driver tends to follow the leader closely to prevent other vehicles cutting in, which causes stronger RI and smaller gap (see Fig. 5). Therefore, to get the desired gap, drivers increase the gap with more discretely driving for ride comfort. 


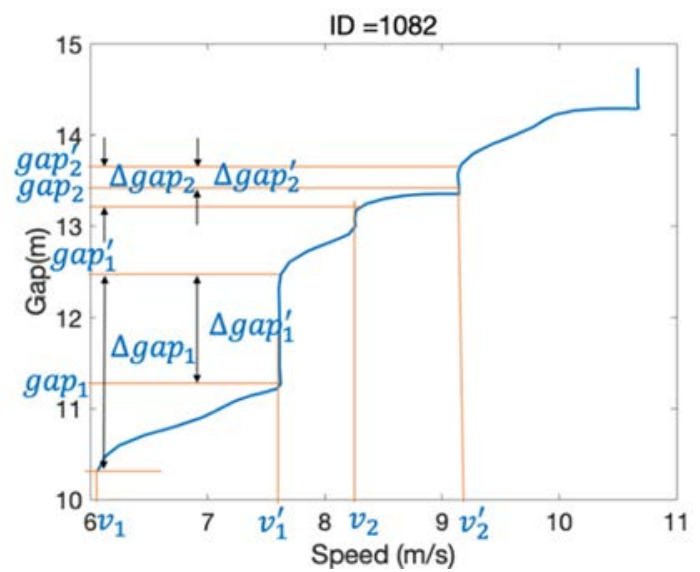

(a)

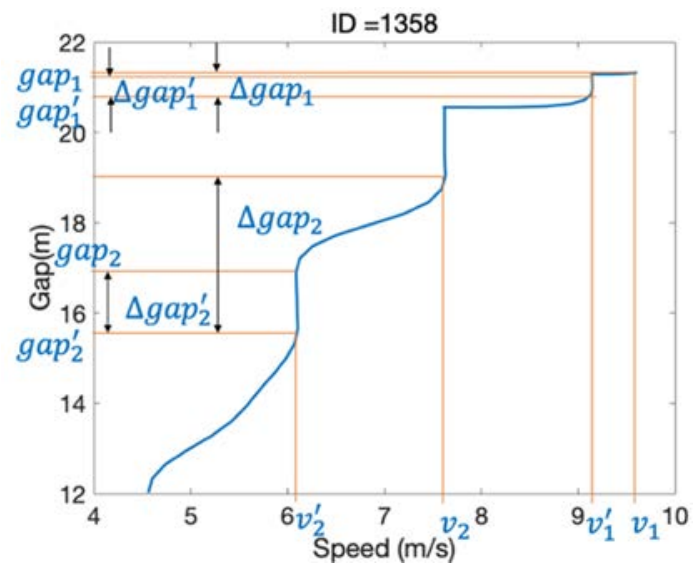

(b)

Figure 5: The relation between $\Delta g a p$ and discrete driving. (a) Acceleration trajectory; and (b) Deceleration trajectory.

Table 8: Correlation between different characteristics.

\begin{tabular}{|l|c|c|}
\hline & Acceleration & Deceleration \\
\hline Response intensity and reaction time & $-0.108(0.115)$ & $-0.035(0.6873)$ \\
\hline Response intensity and discrete intensity & $-0.247^{* *}(0.000)$ & $-0.280^{* *}(0.000)$ \\
\hline Reaction time and discrete intensity & $0.064(0.350)$ & $0.071(0.408)$ \\
\hline
\end{tabular}

\section{CONCLUSIONS}

The asymmetric driving behaviour is one of the most significant human driving behaviours that affect the dynamics of traffic flow. This paper investigates four characteristics of asymmetric driving behaviour at $\mathrm{CF}$ state. To obtain the mechanism of these asymmetric driving behaviours, firstly, the quantitative methods for gap and gap difference, DI and RI 
are proposed while the Newell model is used to estimate driver's RT. Secondly, with these quantitative methods, the asymmetry of driving behaviour is proved using the NGSIM data. For instance, the gap in acceleration is $4.79 \mathrm{~m}$ larger than in deceleration. The mean gap is always lager in acceleration than that in deceleration when $v \leq 12 \mathrm{~m} / \mathrm{s}$. The RT in acceleration is $49.25 \%$ longer than that in deceleration. $14.06 \%$ difference of DI exists between acceleration and deceleration. For the asymmetry of RI which is estimated at each velocity fragment, the largest RI difference between acceleration and deceleration is $236.99 \%$ at $v=12 \mathrm{~m} / \mathrm{s}$. However, the RI in deceleration is not always higher than that in acceleration.

In addition, the correlation analysis between different factors are implemented, which indicate the four asymmetric characteristics are not independent. With paying more attention on safely driving in deceleration process and closely following and discretely driving in acceleration process, the driving behaviour in acceleration is different from that in deceleration. However, close following and safety driving leads to stronger RI, while more discrete driving results in smaller RI, causing that RI in acceleration process are higher than that in deceleration sometimes.

In summary, the four asymmetric characteristics not only exist but also influence each other. In this regard, a CF model that consider the features of asymmetric driving behaviour and their formation mechanism should be studied, which can improve the model performance a lot. This work is on-going.

\section{ACKNOWLEDGEMENTS}

This research is sponsored by the Natural Science Foundation of China (U1764261, 51422812) and the International Science and Technology Cooperation Program of Science and Technology Commission of Shanghai Municipality (16510711400).

\section{REFERENCES}

[1] Newell, G.F., Instability in dense highway traffic: A review. Proceedings of The Second International Symposium on The Theory of Road Traffic Flow, pp. 73-83, 1965.

[2] Yeo, H., Asymmetric microscopic driving behavior theory. $\mathrm{PhD}$ thesis, University of California Transportation Center, 2008.

[3] Wei, D. \& Liu, H., Analysis of asymmetric driving behavior using a self-learning approach. Transportation Research Part B, 47, pp. 1-14, 2013.

[4] Li, L. \& Chen, X.M., Vehicle headway modeling and its inferences in macroscopic/ microscopic traffic flow theory: A survey. Transportation Research Part C: Emerging Technologies, 76, pp. 170-188, 2017.

[5] Huang, X., Sun, J. \& Sun, J., A car-following model considering asymmetric driving behavior based on long short-term memory neural networks. Transportation Research Part C: Emerging Technologies, 95, pp. 346-362, 2018.

[6] Helly, W., Simulation of bottlenecks in single lane traffic flow. Proceedings of the Symposium on Theory of Traffic Flow, pp. 207-238, 1959.

[7] Bando, M., Hasebe, K., Nakayama, A., Shibata, A. \& Sugiyama, Y., Dynamical model of traffic congestion and numerical simulation. Physical Review E, 51(2), pp. 10351042, 1995.

[8] Treiber, M., Kesting, A. \& Helbing, D., Congested traffic states in empirical observations and microscopic simulations. Physical Review E, 62(2), pp. 1805-1824, 2000. 
[9] Gong, H., Liu, H. \& Wang, B.H., An asymmetric full velocity difference car-following model. Physical A: Statistical Mechanics and its Applications, 387(11), pp. 25952602, 2008.

[10] Tordeux, A., Lassarre, S. \& Roussignol, M., An adaptive time gap car-following model. Transportation Research Part B, 44(8-9), pp. 1115-1131, 2010.

[11] Xu, H., Liu, H. \& Gong, H., Modeling the asymmetry in traffic flow (a): Microscopic approach. Applied Mathematical Modelling, 37(22), pp. 9431-9440, 2013.

[12] Hongfei, J., Zhicai, J. \& Xia, L., Factor analysis for choosing input variables of a carfollowing model. WIT Transactions on The Built Environment, vol. 77, WIT Press: Southampton and Boston, pp. 711-720, 2005.

[13] Newell, G.F., A simplified car-following theory: a lower order model. Transportation Research Part B, 36(3), pp. 195-205, 2002.

[14] Ozaki, H., Reaction and anticipation in the car-following behavior. Transportation and Traffic Theory, 12, pp. 349-366, 1993.

[15] Siuhi, S., Parametric study of stimulus-response behavior incorporating vehicle heterogeneity in car-following models. PhD thesis, University of Nevada, Las Vegas, 2009.

[16] Hidas, P., Evaluation and further development of car following models in microscopic traffic simulation. WIT Transactions on The Built Environment, vol. 89, WIT Press: Southampton and Boston, pp. 287-296, 2006.

[17] Lenz, H., Wagner, C. \& Sollacher, R., Multi-anticipative car-following model. The European Physical Journal B: Condensed Matter Complex Systems, 7(2), pp. 331335, 1999.

[18] Next Generation Simulation (NGSIM), Federal Highway Administration (FHWA). https://ops.fhwa.dotgov/trafficanalysistools/ngsim.htm.

[19] Montanino, M. \& Punzo, V., Making NGSIM data usable for studies on traffic flow theory: Multistep method for vehicle trajectory reconstruction. Transportation Research Record, 2390(1), pp. 99-111, 2013.

[20] Welch, G. \& Bishop, G., An introduction to the Kalman filter. University of North Carolina at Chapel Hill, 8, pp. 127-132, 2013.

[21] Li, L., Chen, X. \& Li, Z., Asymmetric stochastic Tau Theory in car-following. Transportation Research Part F: Traffic Psychology and Behaviour, 18, pp. 21-33, 2013.

[22] Ahn, S., Vadlamani, S. \& Laval, J.A., A method to account for non-steady state conditions in measuring traffic hysteresis. Transportation Research Part C, 34, pp. 138-147, 2013.

[23] Zheng, J., Suzuki, K. \& Fujita, M., Car-following behavior with instantaneous drivervehicle reaction delay: A neural-network-based methodology. Transportation Research Part C, 36, pp. 339-351, 2013.

[24] Khodayari, A., Ghaffari, A., Kazemi, R. \& Braunstingl, R., A modified car-following model based on a neural network model of the human driver effects. IEEE Transactions on Systems, Man, and Cybernetics - Part A: Systems and Humans, 42(6), pp. 1440-1449, 2012.

[25] Newell, G.F., Nonlinear effects in the dynamics of car following. Operations Research, 9(2), pp. 209-229, 1961. 